\title{
Modificações na Angulação Coronária Após Implante de Suporte Vascular Bioabsorvível e de Stents de Cromo-Cobalto e Aço Inoxidável
}

\author{
Mateus Veloso e Silva', J. Ribamar Costa Jr. ${ }^{2}$, Alexandre Abizaid ${ }^{3}$, Rodolfo Staico ${ }^{4}$, \\ Danillo Taiguara ${ }^{5}$, Tarcísio Campostrini Borghi Jr. ${ }^{6}$, Ricardo Costa $^{7}$, Daniel Chamié 8 , \\ Amanda G. M. R. Sousa ${ }^{9}$, J. Eduardo Sousa ${ }^{10}$
}

\section{RESUMO}

Introdução: A conformabilidade do stent, definida como a adaptação da prótese à forma natural do vaso, é a principal responsável pelas alterações geométricas produzidas após o implante do dispositivo, sendo influenciada pelo material e pelo desenho do stent. Ela pode ser aferida medindo-se as modificações da curvatura e a angulação do segmento tratado após o implante do stent. O objetivo deste estudo foi comparar as mudanças na angulação coronária após implante do suporte vascular bioabsorvível (SVB) e das plataformas metálicas de cromo-cobalto e aço inoxidável, utilizadas em stents farmacológicos de segunda geração. Métodos: Foram incluídos, nesta análise retrospectiva, 50 pacientes com lesões únicas, de novo, em coronárias nativas, com diâmetro entre 2,5 e $3,5 \mathrm{~mm}$ e extensão de até $23 \mathrm{~mm}$. Vinte e cinco pacientes foram tratados com SVB e 25 pacientes com plataformas metálicas de cromo-cobalto $(\mathrm{n}=12)$ ou de aço inoxidável ( $n=13$ ). A angulação foi medida usando um software de angiografia coronária quantitativa dedicado. Resultados: A angulação do vaso modificou-se significativamente após o implante dos dispositivos. No grupo submetido ao implante de plataformas metálicas, houve maior modificação do ângulo coronário quando comparado ao tratado com SVB (41,6\% vs. $14,9 \% ; P<0,01)$. Quando observado o comportamento do SVB e das diferentes plataformas metálicas, a alteração do ângulo coronário foi maior para as plataformas de aço inoxidável, seguida das plataformas de cromo-cobalto e os SVB $(53,7 \%$ vs. $28,5 \%$ vs. $14,9 \% ; P<0,01)$. Conclusões: Nesta

\section{ABSTRACT}

Changes in Coronary Angulation After Bioresorbable Vascular Scaffold and Cobalt-Chromium and Stainless Steel Stent Implantation

Background: The conformability of the stent, defined as the adaptation of the prosthesis to the natural shape of the vessel, is the major cause of geometrical changes after stenting and is influenced by the stent material and design. It may be assessed by measuring changes in the curvature and the angulation of the treated segment after stent implantation. The objective of this study was to compare changes in coronary angulation after implantation of the bioresorbable vascular scaffold (BVS) and cobalt-chromium and stainless steel metal platforms used in second-generation drug-eluting stents. Methods: In this retrospective analysis, 50 patients with single de novo lesions in native coronary arteries and diameter between 2.5 and $3.5 \mathrm{~mm}$ and length up to $23 \mathrm{~mm}$ were included. Twenty-five patients were treated with BVS and 25 patients were treated with cobalt-chromium $(n=12)$ or stainless steel $(n=13)$ platforms. Angulation was measured using a dedicated quantitative angiography analysis software. Results: Vessel angulation significantly changed after device implantation. In the group submitted to the implantation of metal platforms there was greater coronary angulation change when compared to the group treated with BVS $(41.6 \%$ vs. $14.9 \%$; $\mathrm{P}<0.01)$. When we analyzed the performance of the BVS and the different metal platforms, coronary angulation change was greater for

\footnotetext{
1 Residente do Serviço de Cardiologia Invasiva do Instituto Dante Pazzanese de Cardiologia. São Paulo, SP, Brasil.

${ }^{2}$ Doutor. Cardiologista intervencionista do Serviço de Cardiologia Invasiva do Instituto Dante Pazzanese de Cardiologia. São Paulo, SP, Brasil. ${ }^{3}$ Livre-docente. Diretor da Divisão de Cardiologia Invasiva do Instituto Dante Pazzanese de Cardiologia. São Paulo, SP, Brasil.

${ }^{4}$ Doutor. Cardiologista intervencionista do Serviço de Cardiologia Invasiva do Instituto Dante Pazzanese de Cardiologia. São Paulo, SP, Brasil. 5 Residente do Serviço de Cardiologia Invasiva do Instituto Dante Pazzanese de Cardiologia. São Paulo, SP, Brasil.

${ }^{6}$ Residente do Serviço de Cardiologia Invasiva do Instituto Dante Pazzanese de Cardiologia. São Paulo, SP, Brasil.

${ }^{7}$ Doutor. Cardiologista intervencionista do Serviço de Cardiologia Invasiva do Instituto Dante Pazzanese de Cardiologia. São Paulo, SP, Brasil.
}

\footnotetext{
${ }^{8}$ Cardiologista intervencionista do Serviço de Cardiologia Invasiva do Instituto Dante Pazzanese de Cardiologia. São Paulo, SP, Brasil. ${ }^{9}$ Livre-docente. Diretora Geral do Instituto Dante Pazzanese de Cardiologia. São Paulo, SP, Brasil.

10 Livre-docente. Diretor do Centro de Intervenções em Doenças Estruturais do Coração do Instituto Dante Pazzanese de Cardiologia. São Paulo, SP, Brasil.
}

Correspondência: Mateus Veloso e Silva. Avenida Dante Pazzanese, 500 - Vila Mariana - São Paulo, SP, Brasil - CEP 04012-180 E-mail: mateus veloso@hotmail.com

Recebido em: 15/9/13 • Aceito em: 17/11/13 
avaliação preliminar, o SVB produziu uma menor mudança da angulação coronária. O impacto clínico deste achado necessita ser investigado prospectivamente em uma coorte maior e mais complexa.

DESCRITORES: Vasos coronários. Stents. Implantes absorvíveis. the stainless steel platforms, followed by cobalt-chromium platforms and the BVS $(53.7 \%$ vs. $28.5 \%$ vs. $14.9 \%$; $P<0.01)$. Conclusions: In this preliminary assessment, the BVS produced a smaller coronary angulation change. The clinical impact of this finding must be prospectively investigated in a larger and more complex cohort.

DESCRIPTORS: Coronary vessels. Stents. Absorbable implants.

\section{Dispositivos do estudo}

O SVB ABSORB ${ }^{\circledR}$ é um dispositivo expansível por balão, constituído por um suporte polimérico de ácido poli-L-láctico (PPLA), recoberto por uma fina camada de matriz amorfa de ácido poli-D,L-láctico contendo $100 \mu \mathrm{g} / \mathrm{cm}^{2}$ de everolimus. As hastes do dispositivo têm $150 \mu \mathrm{m}$ de espessura e células com formato de zig-zag, interligadas por três pontes longitudinais. Embora radiotransparente, esse SVB possui uma marcação de platina em cada extremidade, o que permite a visualização angiográfica de seus limites. $\mathrm{O}$ stent $\mathrm{Xience} \mathrm{V}^{\circledR}$ consiste de uma plataforma de cromo-cobalto, com polímero fluorado biocompatível e everolimus na concentração de $100 \mu \mathrm{g} / \mathrm{cm}^{2}$, apresentando uma espessura das hastes de $81 \mu \mathrm{m}$, associada com espessura polimérica de 7,6 $\mu \mathrm{m}$. Já o stent BioMatrix ${ }^{\circledR}$ tem plataforma de aço inoxidável, hastes com espessura de $112 \mu \mathrm{m}$, coberta por um polímero biodegradável de ácido polilático (PLA), contendo Biolimus A9 $(15,6 \mu \mathrm{g} / \mathrm{cm})$.

\section{Procedimento}

As lesões foram tratadas com técnicas habituais de intervenção, que incluíam uma pré-dilatação obrigatória com balão mais curto e com diâmetro $0,5 \mathrm{~mm}$ menor do que o dispositivo utilizado. A pós-dilatação, que era realizada a critério do operador, deveria ser feita com balões não complacentes, pelo menos 30\% mais curtos que o stent ou o SVB implantado.

\section{Análise angiográfica}

Após a administração da nitroglicerina intracoronária, estudos angiográficos em série foram obtidos em duas projeções ortogonais correspondentes, no pré e no pós-procedimento. A análise angiográfica foi realizada off-line por operadores experientes, no Instituto Dante Pazzanese de Cardiologia, com um software validado para análise coronária quantitativa (QAngio XA versão 7.3, Medis, Leiden, Holanda). O diâmetro mínimo do lúmen $(\mathrm{DML})$ e o diâmetro de referência (DR), obtido a partir de uma média dos $5 \mathrm{~mm}$ proximal e distal à lesão alvo, foram usados para calcular o diâmetro da estenose $[\mathrm{DE}=(1-\mathrm{DML} / \mathrm{DR}) \times 100]$. $\mathrm{O}$ ganho agudo foi obtido a partir da diferença do DML pré e pós-procedimento. Para a avaliação de angulação, foi utilizada a projeção angiográfica com o menor encurtamento do 
vaso e a menor sobreposição de imagens. Os ângulos foram obtidos usando uma ferramenta específica, um compasso digital, acoplado ao sistema de análise quantitativa, que foi posicionado dentro do segmento alvo, na altura do DML pré-procedimento. Em seguida, a angulação foi determinada medindo o ângulo interno entre as linhas centradas desenhadas nos segmentos coronários proximal e distal adjacentes ao DML. Os ângulos das lesões foram medidos nas mesmas projeções pré e pós-procedimento, durante a diástole. (Figura)

\section{Análise estatística}

As variáveis categóricas foram apresentadas em números absolutos e porcentuais, enquanto as variáveis contínuas foram descritas como médias e desvios padrão. As variáveis quantitativas foram analisadas por meio do teste não paramétrico de Mann-Whitney (dois grupos) ou teste de Kruskal-Wallis (três grupos). A associação entre as variáveis categóricas foi avaliada pelo teste qui-quadrado ou exato de Fisher. A correlação de Spearman foi utilizada para determinar se havia correlação entre as variáveis angiográficas pré-tratamento (DML, DR, DE, extensão da lesão e ângulo da lesão) e a mudança relativa entre os ângulos antes e após o procedimento.

\section{RESULTADOS}

Foram avaliados 50 pacientes submetidos à angioplastia eletiva, sendo 25 tratados com SVB ABSORB ${ }^{\circledR}$, 12 com o stent de cromo-cobalto (Xience $\mathrm{V}^{\circledR}$ ) e 13 com o stent de aço inoxidável (BioMatrix ${ }^{\circledR}$ ). As características clínicas estão resumidas na Tabela 1. Não houve diferença significativa entre os grupos quanto às características clínicas, com exceção de uma maior prevalência de infarto do miocárdio prévio entre os tratados com stents metálicos.

O vaso alvo mais frequentemente abordado no grupo tratado com SVB foi a artéria descente anterior, enquanto nos tratados com stents metálicos foi a artéria circunflexa $(P=0,09)$. As análises angiográficas pré-procedimento demonstraram não haver diferença entre os grupos em relação às variáveis analisadas, incluindo o diâmetro de referência do vaso tratado e a extensão da lesão. No pós-procedimento, os pacientes tratados com os SVB mostraram menores diâmetros luminais mínimos e ganho agudo comparados aos stents metálicos (Tabela 2).

A angulação do vaso modificou-se significativamente após o implante dos dispositivos (Tabela 3). No grupo submetido ao implante de plataformas metálicas, houve maior modificação relativa do ângulo coronário quando comparado ao tratado com SVB (41,6\% vs. 14,9\%; $P<0,01)$. Quando observado o comportamento do SVB e das diferentes plataformas metálicas, a alteração relativa do ângulo coronário foi maior para a plataforma de aço inoxidável, seguida da plataforma de cromo-cobalto e os SVB $(53,7 \%$ vs. $28,5 \%$ vs. $14,9 \% ; P<0,01)$.

A única variável angiográfica pré-intervenção associada à mudança relativa entre os ângulos, antes e depois do procedimento, foi o ângulo da lesão antes da intervenção $(P<0,01)$, conforme demonstrado na Tabela 4.
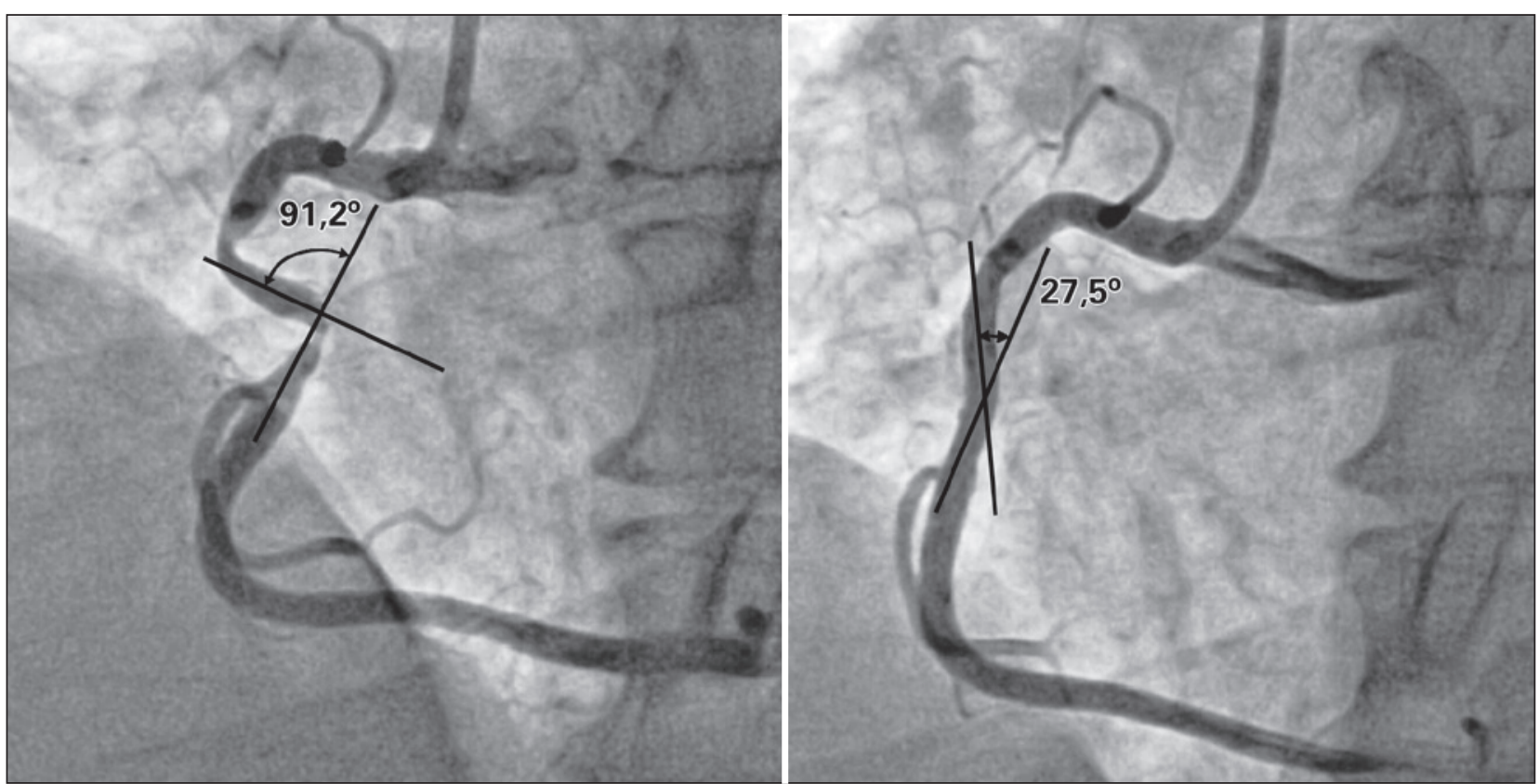

Figura. Análise da angulação coronária. Valores obtidos antes e após o implante de stent, utilizando compasso digital acoplado ao sistema de análise quantitativa. 
TABELA 1

Características clínicas

\begin{tabular}{|c|c|c|c|c|c|c|}
\hline Características & $\begin{array}{c}\text { SVB } \\
(n=25)\end{array}$ & $\begin{array}{c}\text { Plataformas } \\
\text { metálicas } \\
(n=25)\end{array}$ & $\begin{array}{l}\text { Plataforma de } \\
\text { cromo-cobalto } \\
\quad(n=12)\end{array}$ & $\begin{array}{l}\text { Plataforma de } \\
\text { aço inoxidável } \\
\quad(n=13)\end{array}$ & $\begin{array}{l}\text { Valor de } \\
\qquad \mathrm{P}^{\star}\end{array}$ & $\begin{array}{c}\text { Valor de } \\
P^{\star \star}\end{array}$ \\
\hline Idade, anos & $56,8 \pm 7,0$ & $60,4 \pm 8,1$ & $59,8(10,3)$ & $61(4,8)$ & 0,16 & 0,33 \\
\hline Sexo masculino, $n(\%)$ & $15(60)$ & $15(60)$ & $6(50,0)$ & $9(69,2)$ & $>0,99$ & 0,39 \\
\hline Hipertensão arterial, n (\%) & $19(76)$ & $20(80)$ & $9(75)$ & $11(84,6)$ & $>0,99$ & 0,82 \\
\hline Diabetes mellitus, $\mathrm{n}(\%)$ & $5(20)$ & $1(4)$ & 0 & $1(7,7)$ & 0,19 & 0,28 \\
\hline Tabagismo, n (\%) & $3(12)$ & $4(16)$ & $1(8,3)$ & $3(23,1)$ & $>0,99$ & 0,57 \\
\hline Dislipidemia, n (\%) & $19(76)$ & $16(64)$ & $8(66,7)$ & $8(61,5)$ & 0,54 & 0,66 \\
\hline Infarto prévio, n (\%) & $5(20)$ & $15(60)$ & $7(58,3)$ & $8(61,5)$ & $<0,01$ & 0,01 \\
\hline
\end{tabular}

* Comparação entre SVB e stents metálicos; ** comparação entre SVB e plataformas de cromo-cobalto e aço inoxidável.

SVB: suporte vascular bioabsorvivel.

TABELA 2

Características angiográficas e do procedimento

\begin{tabular}{|c|c|c|c|c|c|c|}
\hline Características & $\begin{array}{c}\text { SVB } \\
(n=25)\end{array}$ & $\begin{array}{c}\text { Plataformas } \\
\text { metálicas } \\
(n=25)\end{array}$ & $\begin{array}{l}\text { Plataforama de } \\
\text { cromo- cobalto } \\
(n=12)\end{array}$ & $\begin{array}{l}\text { Plataforama de } \\
\text { aço inoxidável } \\
(n=13)\end{array}$ & $\begin{array}{c}\text { Valor de } \\
P^{*}\end{array}$ & $\begin{array}{c}\text { Valor de } \\
P^{* *}\end{array}$ \\
\hline Vaso alvo, $\mathrm{n}(\%)$ & & & & & 0,09 & 0,24 \\
\hline DA & $13(52)$ & $7(28)$ & $3(25)$ & $4(30,8)$ & & \\
\hline $\mathrm{CX}$ & $4(16)$ & $11(44)$ & $5(41,7)$ & $6(46,2)$ & & \\
\hline \multicolumn{7}{|l|}{ Pré-procedimento } \\
\hline Comprimento da lesão, mm & $11,7 \pm 4,0$ & $11,6 \pm 5,0$ & $10,1 \pm 3,4$ & $12,9 \pm 5,9$ & 0,54 & 0,31 \\
\hline Diâmetro de referência, mm & $2,62 \pm 0,45$ & $2,60 \pm 0,41$ & $2,73 \pm 0,36$ & $2,49 \pm 0,44$ & 0,79 & 0,34 \\
\hline Diâmetro luminal mínimo, mm & $0,87 \pm 0,32$ & $0,84 \pm 0,36$ & $0,90 \pm 0,25$ & $0,78 \pm 0,44$ & 0,73 & 0,52 \\
\hline Diâmetro da estenose, \% & $66,7 \pm 10,5$ & $68,5 \pm 11,2$ & $67,0 \pm 9,2$ & $70,0 \pm 13,0$ & 0,53 & 0,79 \\
\hline Diâmetro luminal mínimo, mm & $2,39 \pm 0,31$ & $2,68 \pm 0,36$ & $2,66 \pm 0,26$ & $2,69 \pm 0,45$ & $<0,01$ & 0,03 \\
\hline Diâmetro da estenose, \% & $8,4 \pm 4,0$ & $6,9 \pm 3,6$ & $8,2 \pm 4,2$ & $5,6 \pm 2,5$ & 0,17 & 0,58 \\
\hline Ganho agudo, mm & $1,51 \pm 0,41$ & $1,83 \pm 0,36$ & $1,76 \pm 0,28$ & $1,9 \pm 0,42$ & $<0,01$ & 0,02 \\
\hline
\end{tabular}

TABELA 3

Modificação dos ângulos dentro e entre os grupos

\begin{tabular}{|c|c|c|c|c|c|}
\hline Dispositivo & $\begin{array}{c}\text { Pré-tratamento } \\
\text { (graus) }\end{array}$ & $\begin{array}{c}\text { Pós-tratamento } \\
\text { (graus) }\end{array}$ & $\begin{array}{c}\text { Mudança absoluta } \\
\text { (graus) }\end{array}$ & $\begin{array}{c}\text { Mudança relativa } \\
(\%)\end{array}$ & Valor de $\mathrm{P}^{*}$ \\
\hline SVB & $16,5 \pm 15,5$ & $13,0 \pm 10,0$ & $3,6 \pm 10,8$ & 14,9 & 0,02 \\
\hline Plataformas metálicas & $20,2 \pm 15,6$ & $11,4 \pm 12,7$ & $8,9 \pm 9,6$ & 41,6 & $<0,01$ \\
\hline Valor de $\mathrm{P}^{\star \star *}$ & 0,22 & 0,32 & $<0,01$ & $<0,01$ & \\
\hline SVB & $16,5 \pm 15,6$ & $13,0 \pm 10,0$ & $3,6 \pm 10,8$ & 14,9 & 0,02 \\
\hline Plataforma de aço inoxidável & $23,0 \pm 14,2$ & $12,9 \pm 13,9$ & $10,1 \pm 7,4$ & 53,7 & $<0,01$ \\
\hline Valor de $\mathrm{P}^{\star \star}$ & 0,14 & 0,49 & $<0,01$ & $<0,01$ & \\
\hline
\end{tabular}


TABELA 4

Correlação entre variáveis angiográficas pré-tratamento e mudança relativa entre os ângulos antes e depois do procedimento

\begin{tabular}{lcc}
\hline Variável & Correlação & Valor de $\mathbf{P}$ \\
\hline Comprimento da lesão & 0,13 & 0,38 \\
Diâmetro de referência & 0,24 & 0,88 \\
Diâmetro luminal mínimo & 0,12 & 0,43 \\
Diâmetro da estenose & 0,04 & 0,77 \\
Ângulo da lesão & 0,68 & $<0,01$ \\
\hline
\end{tabular}

\section{DISCUSSÃO}

Observamos, neste estudo, que tanto as plataformas metálicas quanto o SVB modificaram significantemente a angulação da lesão após o procedimento. Além disso, a menor modificação do ângulo ocorre com os SVB, seguidos das plataformas de cromo-cobalto e das plataformas de aço inoxidável. A única variável angiográfica associada à mudança relativa entre os ângulos foi o ângulo da lesão antes da intervenção.

A eficácia e a segurança dos stents dependem, em parte, de suas características mecânicas. Estudo prévio, que avaliou as propriedades mecânicas de 17 stents metálicos, analisou a força de tração entre tortuosidades, flexibilidade e conformabilidade, e mostrou que essas propriedades dependem do desenho e do material do stent. ${ }^{7}$ A comparação da performance mecânica de sete stents farmacológicos mostrou diferenças entre as diversas plataformas, com o stent BioMatrix ${ }^{\circledR}$ mostrando menor flexibilidade que o Xience $V^{\circledR}\left(30,06 \mathrm{~N} / \mathrm{mm}^{2}\right.$ e $25,78 \mathrm{~N} / \mathrm{mm}^{2}$, respectivamente). ${ }^{4}$ Outro estudo que avaliou a conformabilidade dos SVB e do stent Xience $\mathrm{V}^{\circledR}$ mostrou vantagem para a plataforma bioabsorvível, que demonstrou alterar menos a curvatura e a angulação do vaso tratado. ${ }^{6}$

Vasos curvos têm uma maior associação com o fluxo turbulento e distribuições não uniformes do estresse de cisalhamento, com a curvatura exterior do vaso submetida a um estresse de cisalhamento maior que a curvatura interna. Regiões com baixo estresse de cisaIhamento têm sido associadas com o desenvolvimento de hiperplasia neointimal mais espessa, tanto em stents não farmacológicos ${ }^{2}$ como com os farmacológicos. ${ }^{8}$ O seguimento de 1 ano dos SVB mostrou restauração da anatomia coronária próxima à observada antes do procedimento, e com provável restabelecimento de um padrão mais fisiológico do fluxo sanguíneo e estresse de cisalhamento. ${ }^{9}$

Estudo com pacientes tratados com stents não farmacológicos demonstrou que a localização da lesão-alvo em segmento coronário com ângulo $>33,5^{\circ}$, bem como a alteração na angulação coronária $>9,1^{\circ}$ após o implante dos dispositivos, foi preditora independente de eventos cardíacos maiores e reestenose. ${ }^{10}$ Por outro lado, estudo com 289 pacientes tratados com stents metálicos farmacológicos não conseguiu demostrar associação entre a angulação coronária ou a modificação na geometria vascular com a ocorrência de eventos adversos (falência do vaso alvo, revascularização do vaso alvo ou infarto intra-hospitalar), após 1 ano de seguimento. ${ }^{11}$

As novas gerações de stents farmacológicos, com ligas metálicas mais flexíveis e plataformas mais delgadas e com melhor conformabilidade, têm a possibilidade de alterar menos a geometria coronária que os stents mais antigos. Ainda que os SVB sejam superiores aos atuais stents metálicos em conformabilidade, e por essas diferenças serem tão sutis, é necessário aguardar avaliação de casuísticas maiores e com acompanhamento de longo prazo, para se verificar a tradução clínica desses achados.

\section{Limitações}

Ressaltam-se, como principais limitações deste estudo, seu desenho não randomizado, a pequena população incluída em cada grupo e o perfil de complexidade limitado das lesões.

\section{CONCLUSÕES}

Nesta avaliação preliminar, o implante do suportes vasculares bioabsorvíveis com eluição de everolimus demonstrou alterar em menor grau a angulação coronária comparada às plataformas de cromo-cobalto e de aço inoxidável. O impacto clínico desse achado ainda é incerto e necessita ser investigado prospectivamente, em uma coorte maior e mais complexa.

\section{CONFLITO DE INTERESSES}

Os autores declaram não haver conflito de interesses relacionado a este manuscrito.

\section{REFERÊNCIAS}

1. Wentzel JJ, Whelan DM, van der Giessen WJ, van Beusekom HM, Andhyiswara I, Serruys PW, et al. Coronary stent implantation changes 3-D vessel geometry and 3-D shear stress distribution. J Biomech. 2000;33(10):1287-95.

2. Wentzel JJ, Krams R, Schuurbiers JC, Oomen JA, Kloet J, van Der Giessen WJ, et al. Relationship between neointimal thickness and shear stress after Wallstent implantation in human coronary arteries. Circulation. 2001;103(13):1740-5.

3. Colombo A, Stankovic G, Moses JW. Selection of coronary stents. J Am Coll Cardiol. 2002;40(6):1021-33.

4. Schmidt W, Lanzer P, Behrens P, Topoleski LD, Schmitz KP. A comparison of the mechanical performance characteristics of seven drug-eluting stent systems. Catheter Cardiovasc Interv. 2009;73(3):350-60.

5. Sangiorgi G, Melzi G, Agostoni P, Cola C, Clementi F, Romitelli $P$, et al. Engineering aspects of stents design and their translation into clinical practice. Ann Ist Super Sanita. 2007; 43(1):89-100. 
6. Gomez-Lara J, Garcia-Garcia HM, Onuma Y, Garg S, Regar E, De Bruyne B, et al. A comparison of the conformability of everolimus-eluting bioresorbable vascular scaffolds to metal platform coronary stents. JACC Cardiovasc Interv. 2010;3(11):1190-8.

7. Rieu R, Barragan P, Garitey V, Roquebert PO, Fuseri J, Commeau $\mathrm{P}$, et al. Assessment of the trackability, flexibility, and conformability of coronary stents: a comparative analysis. Catheter Cardiovasc Interv. 2003;59(4):496-503.

8. Gijsen FJ, Oortman RM, Wentzel JJ, Schuurbiers JC, Tanabe $\mathrm{K}$, Degertekin $\mathrm{M}$, et al. Usefulness of shear stress pattern in predicting neointima distribution in sirolimus-eluting stents in coronary arteries. Am J Cardiol. 2003;92(11):1325-8.

9. Gomez-Lara J, Brugaletta S, Farooq V, van Geuns RJ, De Bruyne
B, Windecker S, et al. Angiographic geometric changes of the lumen arterial wall after bioresorbable vascular scaffolds and metallic platform stents at 1-year follow-up. JACC Cardiovasc Interv. 2011;4(7):789-99.

10. Gyongyosi M, Yang P, Khorsand A, Glogar D; Austrian Wiktor Stent Study Group and European Paragon Stent Investigators. Longitudinal straightening effect of stents is an additional predictor for major adverse cardiac events. J Am Coll Cardiol. 2000;35(6):1580-9.

11. Gomez-Lara J, Heo JH, Brugaletta S, Garg S, Garcia-Garcia HM, van Geuns RJ, et al. Risk of target lesion failure in relationship to vessel angiographic geometry and stent conformability using the second generation of drug-eluting stents. Am Heart J. 2011;162(6):1069-79. 